\title{
Virtual Planning and Intraoperative Navigation in Craniomaxillofacial Surgery
}

\author{
J. Guiñales, J.L. Cebrian*, M. Burgueño \\ Servicio de Cirugía Oral y Maxilofacial, Hospital Universitario La Paz, Madrid (Spain) \\ Received 16 September 2016 | Accepted 1 November 2016 | Published 23 December 2016
}

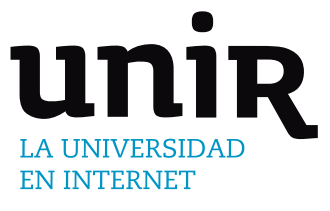

KEYWORDS

Craniomaxillofacial

Surgery, Facial

Reconstructive Surgery, Computer-assisted

Surgery, Surgical

Navigation, Surgical

Planning.

DOI: $10.9781 /$ ijimai.2017.4512

\section{INTRODUCTION}

$\mathrm{T}$ collaboration between engineers and surgeons during the last ten years has facilitated the development of several tools that have incredibly improved the predictability and the results of the surgeries.

Virtual planning or surgery planning assisted by computer allows us to pre do the surgery, reproducing our mental plan, by working over a virtual 3D model of the patient obtained through a CT (computer tomography). Once we have the CT of the patient, there are several different softwares that can create this $3 \mathrm{D}$ model and with different tools, depending on the software, enable us to a wide range of options: select the affected area, segmentation of the skeleton in the different bones, perform osteotomies, move the bones, make mirroring images of the unaffected side, import STL models of the prostheses or plates or pre simulate the soft tissue changes after the surgery. Virtual planning represents the main example of the collaboration between surgeons and engineers. During this step, both will work hand by hand designing the surgical plan with the common goal of achieving the highest precision.

However, surgical planning is just one of the examples of this collaboration. Once we have finished our virtual planning we can also impress stereolithographic models of the different anatomical structures, the plates and prostheses or guides for the osteotomies than will help us through the different steps of the surgery.

One of the last applications resulting from this engineer-surgeon collaborations is the surgical navigation. Surgical navigation was born as an evolution of the stereotactic surgery in the field of Neurosurgery due to the integration of both the intraoperative positioning and the imaging diagnostic systems. Thanks to this tool we can intraoperatively know our real position by placing a pointer on the patient that will shows us precisely the situation in the patient's CT. This will help us while we are working with deep structures and more importantly in those cases where important structures such as arteries and nerves are in the nearby

\footnotetext{
* Corresponding author.

E-mail address: josel.cebrian@salud.madrid.org
}

of our surgical field and must be protected. But the surgical navigation also allows us to check if we are following accurately the surgical plan. For example if we have placed a prosthesis, during the virtual planning step, in an specific position, we can check during the surgery if the real position fits with the planned one, and if not, correct it.

Although all these tools have implemented all kind of surgical specialities, they have become indispensable in the field of reconstructive surgery and specially facial reconstructive surgery. In this field every millimetre counts and it can be the difference between a good result and a complete disaster. We, as craniomaxillofacial surgeons, work in a field with a complex spacial relationship between the different anatomical structures in which these new technologies have revolutioned the way we plan and treat our patients.

In this last article, we want to share with you two different cases in which we will be able to show the advantages of these new tools in the craniomaxillofacial surgery.

\section{CAses}

First of all we will go through a simple case that we can came across frequently in our daily practice. This is the case of orbital fractures. Patients often suffer these kind of fractures resulting from direct trauma to the eye globe. Usually the floor of the orbit is the most affected wall, followed by the medial wall, with combining fractures in up to $50 \%$ of the cases.

The orbital cavity has an inverted pyramid form, with the base of this pyramid located at the anterior part. This cavity accommodates the eye globe, ocular muscles, orbital fat, nerves and vessels. Any change in the position of the walls will have a direct impact in the orbital volume and so it will affect to the eye position with an aesthetic and functional result that can lead to diplopia (double vision), exofalmos (anterior eye displacement), enoftalmos (posterior displacement of the eye), dystopia (vertical displacement of the eye) and even vision impairment resulting from orbital nerve affection. In such cases, reconstruction of the affected wall must be accomplished in order to restore the normal position of the wall and the orbital volume. 
Until virtual planning softwares and navigations systems appeared, these cases were treated having as the only guide the preoperative $\mathrm{x}$-rays of the patient. In such situations the surgeon can only guess the theoretical situation in which the affected wall should be, without any intraoperative references except for the surrounding structures that in many cases are also affected. Today, virtual planning software allows us to perform previously this surgery on the computer working with a 3D model of the orbit and plan the exact position in which the affected wall should be. Thanks to mirror image tools, we can reconstruct the orbit based on the unaffected side. As we know, orbits usually are symmetric in form and volume in non-syndromic patients, so we can make a mirror image of the unaffected side as use it as a model for the reconstruction. As so, we can design the desired position of the new wall, we can measure the dimensions of the fracture and now in advance the size and form of the mesh we will need to reconstruct it, we can now the spacial relations with the adjacent structures such as the optical nerve, we can measure the orbital volume of the new orbit and compare it with the healthy side, we can use the STL models of the commercialised meshes and see how the fit into the defect or if we will need to customize them, and if so do it before going to the operating room and finally we can work with the engineers in designing an specific implant for every case (Fig.1 and 2).

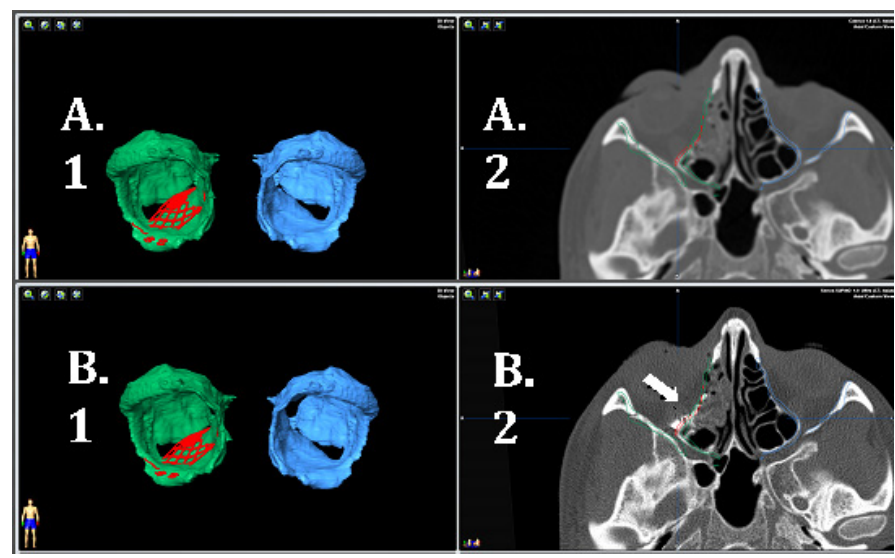

Fig. 1. A.1. 3D reconstruction of the normal orbital cavity. A.2. Medial orbital wall fracture (white arrow). Non-fractured medial orbital wall (red arrow). B.1. 3D orbital reconstruction (green). Mirroring from the healthy orbit (blue).

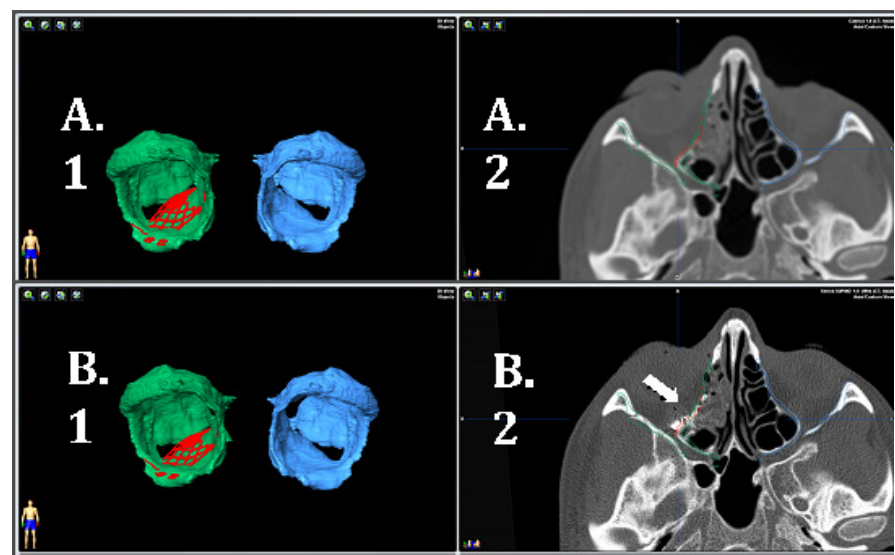

Fig. 2. A.1-2. Orbital reconstruction plate (red), obtained from STL archives of custom made plate, reconstructing orbital walls and placed in the right position using virtual surgery. B.1. Virtual surgical plan. B.2.Post-operative CT scan. Reconstruction plate placed in the right position (white arrow) using navigation

The next step in the orbital reconstruction planning is to use the intraoperative navigation to confirm our surgical planning. First of all we will use navigation to confirm the limits of the fracture and the correlation between the intraoperative findings and the scanner. We can also use it to know the exact point in which we are while we are performing the orbital dissection and to be sure of having a safety distance with the optical nerve. Once we have identified completely the fracture, we will place our orbital mesh and we will use the navigation to be sure that the final position fits with the desired plan. The navigation system incorporates a computer screen in which we can see not only the previous CT but also superimpose the surgical plan so we can in every moment be sure that we are following it faithfully.

The second case that we want to share with you is regarding about the utility of this tools in oncological patients. This is a case of a 64 years old man presenting at our office with a left zygomatic mass of three months of evolution with progressive growth. As the only previous disease, he suffered an adenoid cystic carcinoma 8 years before affecting the buccal mucosa of his left side. He went trough intervention with complete extirpation of the tumor and no risk factors associated. He remained out of clinical or radiological signs of recurrence until the moment he came to our office.

We perform a CT which showed a growing mass affecting to the left zygoma and extending into the maxillary bone and the orbital floor, with soft tissue extension up to the subcutaneous tissue. An intraoral biopsy confirmed the diagnosis of adenoid cystic carcinoma. A MRI (magnetic resonance imaging) was made for better study of the soft tissue affection.

For the virtual planning we use IPlan from Brainlab. We introduce both the CT and the MRI and thanks to the fusion tool, the software automatically fused both studies so we can work with both at the same time, having great definitions of the bone and the soft tissues. The next step is to define the tumor in the images and for so, the IPlan offers a special tool called Smartbrush that automatically outlines the tumor volume just by selecting the tumor, with a click of mouse, in two slices with different orientations (Figure 3). This tool recognises the selected structure, in this case the tumor, by choosing all the pixels with the same Hounsfield units. Once the tumor is automatically outlined, we can manually improve the selection contouring it with the mouse.

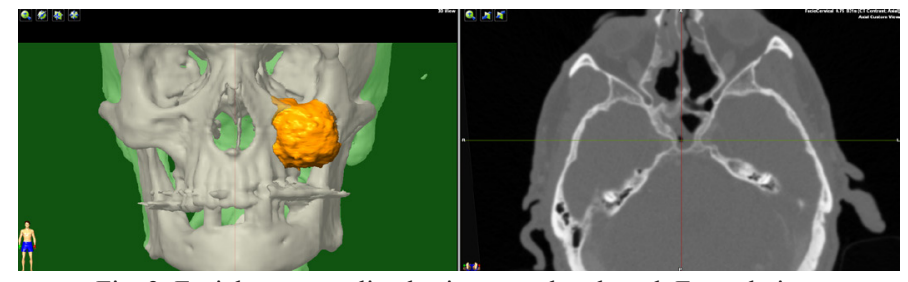

Fig. 3. Facial tumor outlined using smartbrush tool. Frontal view.

Adenoid cystic carcinoma is a malignant tumor that must be resected with free margins in order to ensure a complete resection and avoid recurrence. For that instance, we can easily defined the desired margin with the software just by selecting the amount it, and automatically a new object will be created which will include the tumor and the free selected volume around it (Figure 4). This new object will be our resection piece and the software will show us how it will affect to the adjacent structures. We can also subtract the resection from the 3D reconstruction and see how the final defect will be in order to plan the reconstruction. In our case, the resection will include the zygoma, the left maxilla including the alveolar process and the teeth up to the canine but preserving the nasal buttress, the orbital floor and infraorbital rim and the overlying skin. 


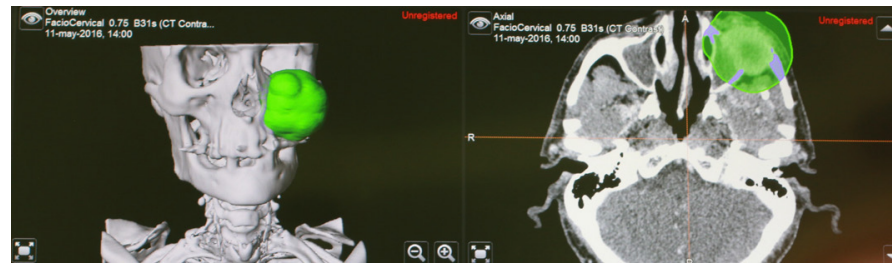

Fig. 4. Planned surgical resection with security margin (green).

After finishing the virtual planning we arrange an online appointment with the engineer of the brand that will provide us the surgical prosthesis. Previously we sent him the STL of the plan so he can have in advanced how our resection will be. In this case we worked with Johnson \& Johnson which at the same time works with Materialise for all the stuff regarding the individualised implants and surgical guides. During this online appointment, we reviewed the surgical plan and we designed, the cutting guides with dental and bone anchorage that will marked the desire resection (Figure 5). Although we can navigate the resection following the plan without guides, these will help us to be more accurate and have a better fit of the prosthesis. After this, we designed the prosthesis, in this case a titanium customised implant that will reconstruct the orbital floor, the infraorbital rim, the zygoma and the anterior wall of the maxilla. Once again, we used a mirror image tool to superimpose the unaffected side over the affected side without the resection, so we will keep the symmetry during the reconstruction. We also planned the drilling holes in the prosthesis were we will place the screws to fix it and two guide lines over the orbital floor of the implant, in order to be able to navigate it and have a double check of correct position (Figure 6).

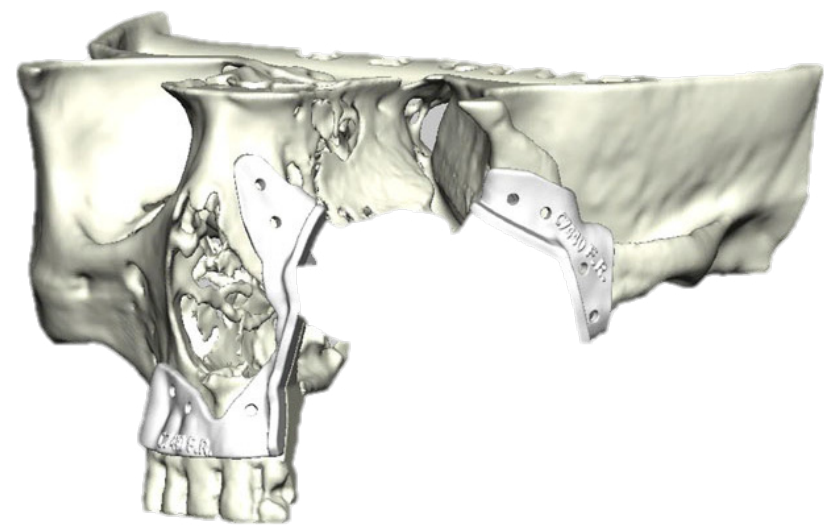

Fig. 5. Cutting guides (green arrow) and defect following resection (red arrow).

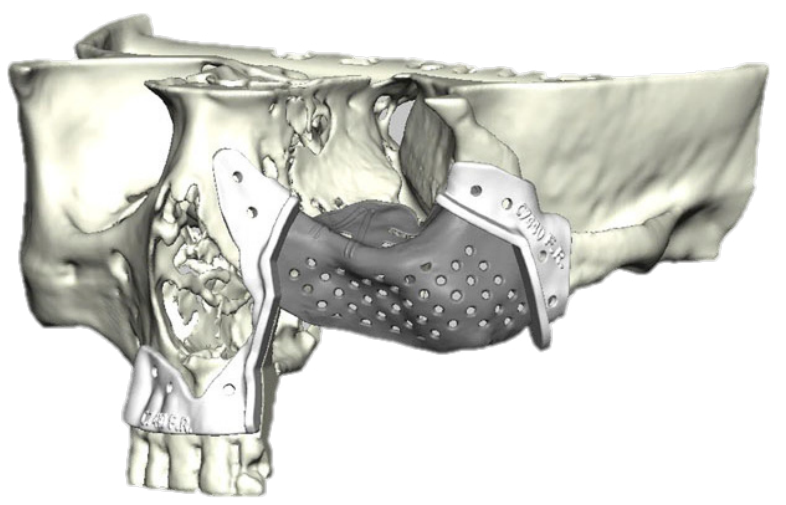

Fig. 6. Customized prostheses.

As you can see through the intraoperative pictures, thanks to the virtual planning, the surgical guides and the navigation system, in every moment we were sure that we were perfectly fitting the plan, with no option to fail, and achieving the greatest accuracy (Figure 7).

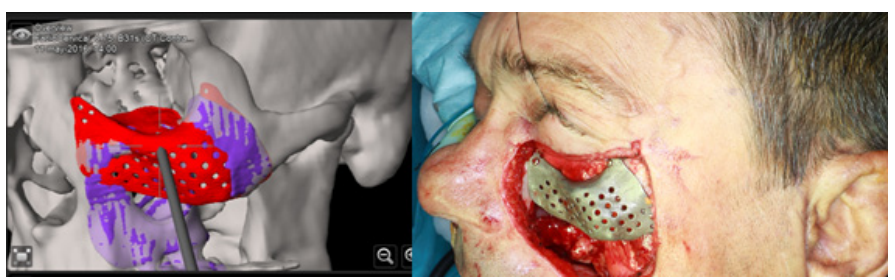

Fig. 7. Introperative navigation and surgical picture showing the accuracy of plate placement.

In conclusion, the new tools emerging from the collaboration between engineers and surgeons, make us no better surgeons but more predictable surgeons which at the end will help us to reach our final goal, offer to the patient the best treatment option.

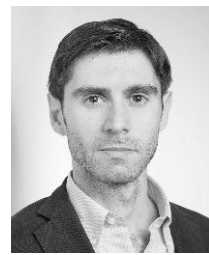

\section{Jorge Guiñales}

Bachelor of Medicine and Surgery from the Autonomous University of Madrid. Resident of Oral \& Maxillofacial Surgery at the University Hospital La Paz, Madrid. Dentistry student at the European University of Madrid. Vocal residents SECOM.

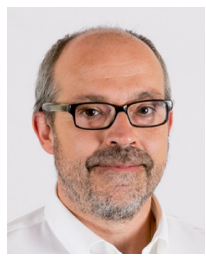

\section{José Luis Cebrián Carretero}

José Luis Cebrián Carretero got his Medical Degree Universidad Autónoma de Madrid (UAM) in 1993. He is $\mathrm{PhD}$ by the same university in 2005 . He also studied Oral and Maxillofacial training H.U. La Paz from 1995 to 2000. He got is Dental Degree in the Universidad Europea de Madrid (UEM ) in 2009. He is fellow of the European Board of Oral and Maxillofacial Surgery, Specialist in Oral and Maxillofacial Surgery, La Paz University Hospital, Madrid and Chief of Division in Oral and Maxillofacial Surgery, La Paz University Hospital, Madrid, and Director of Oral and Maxillofacial Surgery Departments at Quironsalud Hospital Sur de Madrid y Clínica La Luz. Finally he is Vice-President of the SECOM (Spanish Society of Oral and Maxillofacial Surgery). He is author of 2 books, 15 chapters and more than 50 scientific articles. His main research areas are Implantology, Head and Neck Oncologic Surgery and Orthognatic and Oral Surgery.

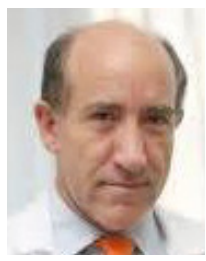

Miguel Burgueño García

Bachelor of Medicine and Surgery from the Complutense University of Madrid. Trained as a specialist in oral and maxillofacial surgery at the Hospital La Paz, where he served as deputy until 2005, when it becomes Chief. Degree of Doctor of Medicine from the Autonomous University of Madrid in 1995 and recognized career in facial surgery, in the following areas: reconstructive, microsurgical techniques, orthognathic surgery, deformities, facial fractures and facial cosmetic surgery. Associate professor in the Department of Surgery at the Autonomous University of Madrid. Director of numerous specialized training courses. He currently manage the course of Implantology at the Autonomous University of Madrid. European Board in Oral and Maxillofacial Surgery (certificate of excellence for professionals in the European Union). President of the Spanish Society of Oral and Maxillofacial Surgery between 2005-2007. 